\title{
Extended Enterprise Communications Based On The Client/Server Paradigm
}

\author{
Marcelo L. Martin ${ }^{l}$, Manuel M. Barata ${ }^{2}$ Eduardo Zavalla \\ ${ }^{1}$ Instituto de Automática - Facultad de Ingeniería \\ Universidad Nacional de San Juan \\ Av. San Martin - oeste - 1109 - 5400 San Juan - Argentina \\ mmartin@inaut.unsj.edu.ar \\ ${ }^{2}$ ESTEC - Estudos e Tecnologias da Informação, Lda. \\ Taguspark, Edificios Tecnologias I N. 21, 2780 Oeiras - \\ Portugal,mmb@estec.pt
}

\begin{abstract}
This paper presents a distributed computing architecture based on the client-server paradigm for extended enterprise information flow management. A computing architecture capable of binding all member companies' internal computing systems and dealing with heterogeneous information technology systems is proposed. This scheme should integrate the required information for enterprise specific tasks for resources optimisation. The use of available local area networks and standardised protocols for data retrieval and transportation is encouraged.
\end{abstract}

Keywords

Extended enterprise, Client-server paradigm, Internet protocols, Intranets

\section{INTRODUCTION}

The term "Extended Enterprise" is applied when different product functionalities are provided separately by different companies (Browne, 1996). Present concepts such as market globalisation and outsourcing must be taken into account for product commercialisation in an extremely competitive and dynamic market. 
Even when the extended enterprise model has important economic as well as functional advantages, it usually implies redesigning the administrative structure, and sometimes, the productive structure of the members. To unify all those efforts oriented to a common goal in an optimised fashion, a robust and flexible communication link is required. This link should be the main way for internal and public document transfer among all companies and people who build up the productive chain.

Administrative and productive areas nowadays are typically aided by computers with software which could manage all the information needed to "conglomerate" the various enterprises into a single entity: the extended enterprise.

However, the main drawback of this scheme lies in the heterogeneity of the companies software packages and hardware platforms which are tuned to their specific needs, turning their linkability into a rather difficult task. This incompatibility may result from:

- Proprietary software packages.

- Inherited hardware architectures.

- Outdated programming languages and hardware platforms.

- Lack of standards.

Nevertheless, lower prices of computers and the availability of the state-of-the-art software technology allows incorporating the following facilities in almost any company:

- Business information aided by computers.

- Local area networks, even in the smallest company.

- Telephone and Internet connectivity.

This paper presents the analysis of a client-server architecture which allows many users who are geographically spreaded to access to specific information related to joint-project. The re-utilisation of any computer system architecture available at the company is encouraged, and the additional equipment necessary for information interchange is analysed.

\section{EXTENDED ENTERPRISE STRUCTURE}

An extended enterprise is defined as a set of companies which are totally or partially related in order to cooperate for a common business goal. From an external point of view, this integration can be seen as a single commercial block.

At present, this kind of structure, usually called Extended Enterprise or Virtual Enterprise (Camarinha-Matos L.M., Afsarmanesh H, et al., 1997 ; Camarinha- 
Matos L.M., Carelli R. et al., 1997), is becoming ubiquitous in different industrial areas.

A specific area, is the food industry where studies about the formalisation of "bindings" among all partners have been carried out. For example, the SCM+ project (Beyond Supply Chain Management in Food Industry) (SCM+, 1997), presents interesting results on productive chain modelling, taking into account several aspects such as the kind of the company involved and communications frequency among all member companies.

The actors involved in the supply chain are: farmers, commodities suppliers, collecting centres, production centres, customers and services. Regarding the information exchange process, the initiative is taken accordingly to each situation.

These actors, most probably, own communications means with different technological levels, ranging from conventional mail to electronic mail. However, the experience has shown that electronic communications provides the most reliable and quickest access and response, permitting to avoid delays in purchase orders, to overcome temporary incapacity of a supplier, and other problems.

As a conclusion, all members of an extended enterprise should tend to be linked by a low cost platform and an independent standardised communication system. The implementation of a truly Just-In-Time philosophy requires an infrastructure that supports a stronger client-supplier interaction.

\section{PRELIMINARY REQUIREMENTS}

The majority of the so called "small companies", which have a relevant role in the extended enterprise model, has proved not to be "computing resources integrators". That is, their computer systems and software have not been developed neither to co-operate with another system nor to carry out remote input/output tasks. This means that heterogeneous data formats must be shared among the group members.

By adding standard data connectivity software and network protocols such as ODBC and TCP/IP-related stack the above drawback can be overcome.

Standards are the key factor in extended enterprise data sharing. Several companies will be sending data back and forth to each other, and this data flow must be available immediately to whom may need it, without any need of data conversion.

Even though there are many emerging standards and models for data management, only those already extensively tested should be used. This will enable to build an updateable system, expandable with well known technology future support. In this 
way, a lot of hardware platforms will be supported, so vendors independence is assured.

\section{CLIENT-SERVER ARCHITECTURE}

Trying to move every user towards standards, all at once, is expensive and not recommended. Related costs can be considerable without any significative benefit in the short run.

However, a statistical analysis of the company information flow shows that only a reduced amount of data is really interesting to other users in the chain. Having these data available in a secure place and allowing only qualified users to access them is the best way to start with.

This behaviour can be thought as a server giving access to resources (data) which can be retrieved by several clients (users). This model is known as the client-server paradigm (Figure 1).

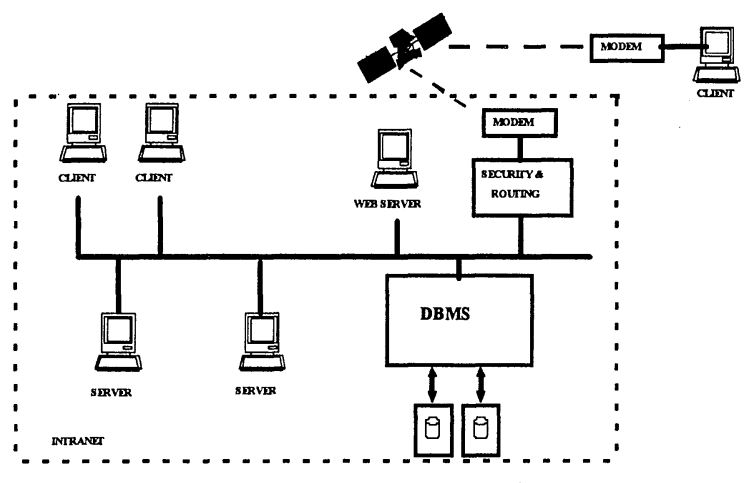

Figure 1 Proposed client/server architecture.

Again, standard protocols and database connectivity must be used in order to assure an uniform way for information management, but they should be applied only to those data that have proved to be useful for all member companies. In this model, database access standards can be followed only at server level, having clients to use only network protocols standards.

A valid choice for this network protocol is the TCP/IP stack. This protocol has been used from the early sixties and has proven to be a de-facto standard for reliable data transfer over the world. Furthermore, it is the protocol currently used by Internet, so by using it, this widely spreaded network will allow data sharing among enterprises located in different countries. 
A TCP socket establishes a virtual channel between a server and a client independently of their hardware and operating system types. However this virtual channel does not define any special structure to the transmitted/received data. They only assure an error-free byte-stream communication channel. Here is where the high level service protocol appears, e.g. simple mail transfer protocol (SMTP), hypertext transport protocol (HTTP), for structuring the application specific messages. By defining the application specific protocol, it is possible to operate clients spreaded world-wide and connected through the Internet.

In this context a client becomes a running computer program that can be either:

- An user interface browser, or

- A specific application, such as a data base entry.

There are two types of connections between server and client:

- Remotely supported by modem or by a connection through Internet.

- Locally through the local area network (LAN) implemented by conventional infrastructures.

The implementation of this model requires the basic TCP/IP services for establishing the virtual communication channels, and proper application protocols, like those of Table 1.

Table 1 Level implementation details

\begin{tabular}{lll} 
LEVEL & INTERFACE & PROTOCOL \\
\hline Remote Client & Browser & HTML and other \\
Local Client & Browser & HTML and other \\
Data Server & Data Base Server & CGI or other \\
WEB Server & HTTP Server & HTML \\
\hline
\end{tabular}

To implement the above architecture, its advantages and drawbacks must be analysed, taking into the same level of consideration the practical, economical and technological factors.

\section{A CLIENT-SERVER NETWORK IMPLEMENTATION}

A private network based on Internet protocols (from the TCP/IP stack) is called an intranet. The intranet model relies on using an existing enterprise network for 
information interchange by adding Internet-like capabilities. This configuration enables to take advantage from various software tools widely tested (and most of them free of charge) to make data available and to perform data retrieval.

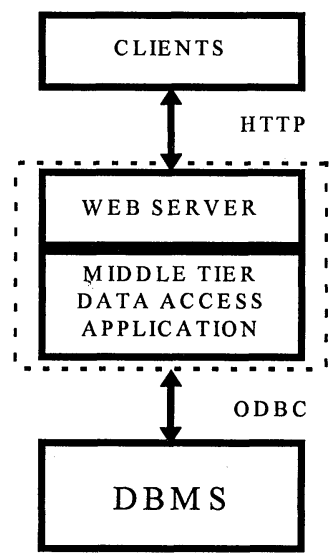

Figure 2 Three tier client/server model.

Since HTTP protocol has become a standard, data access is typically done by an HTTP server (also called a WEB server) and complementary, an HTML client browser. However, data servers are allowed to stay either in the same station as the WEB server or in another station over the network. A third application other than the WEB server and the data server is responsible for data transfer between them upon user requests. This kind of structure is expected to be found in intranets linking inherited databases not developed for remote access. It is called a three tier client-server model (Figure 2)

Many advantages can be provided by an intranet, namely :

- Small initial investment, provided a LAN structure is available.

- Efficient utilisation of computational resources in a distributed architecture.

- Only an HTML browser is necessary as user interface.

- Almost no learning curve for users.

- Scalability.

- Multimedia support.

- No connection to Internet is required.

- Hardware and software independence.

- Communications costs lower than telephone rates, provide Internet is used as a carrier.

Weakness may be traced to: 
Security: Concerning security, three aspects must be taken under consideration: privacy assurance, intruder avoidance and authentication.

Privacy assurance means that all data transferred over the Internet be inaccessible to undesired users. This is a difficult problem to be solved because of the public nature of Internet: somewhere in the communication path the data might be traced by someone else. The actual remedy for this problem is to use encryption techniques like DES, RSA and similar ones. However these techniques rely on passwords and all the privacy can be broken if the password is known by someone else.

Intruder avoidance deals with the problem of avoiding undesired or without access permission users to establish communication with server and computers accessible through the Internet. Firewall technology is the current solution to this problem (Cheswick and Belloin, 1994). From its application the concept of intranet emerged, i.e. the internal protected network. Basically this method examines all incoming network packages and validates their access rights to the internal network, namely the access to the services' servers. The weak point of firewall arises whenever internal users establish external communications through local modems and phone circuits to other external unprotected LANs, thus the firewall is bypassed and a "security hole" is created.

Finally, authentication constitutes the third problem to solve. It consists on the validation of received messages from the Internet. Suppose for instance that an invoice message arrives to a production factory. How can the factory personnel decide if the message has been sent by the right sender or by an impostor? The current solution for this problem is through encryption based on public and private keys. The sender encrypts the message with its private key (only he knows it) and the receiver decrypts the message using the public key (known by everybody). A practical application of this method is commonly called the digital signature of messages.

Again the weak point of this technique relies on the confidentiality of the private key and the availability of a public key directory.

Initial network structure: If a LAN is not available, intranet implementation costs would be high. A financial analysis is mandatory in order to estimate associated investments of recovery time and convenience.

However, if a LAN is already set up, the intranet is easily obtained by installing a WEB server, a firewall and the middle layer application. Depending on the kind of hardware and protection scheme, the implementation cost varies, but usually it can be considered of a relatively low cost. 
Communications delays: Truly real time data transfer is impossible because access times and execution speed are strongly related to network load. Intranet times are expected to be lower (milliseconds to minutes) than Internet times (from minutes to hours). Anyway, no data transfer service between companies seems to need a realtime approach, as long as the involved time is acceptable.

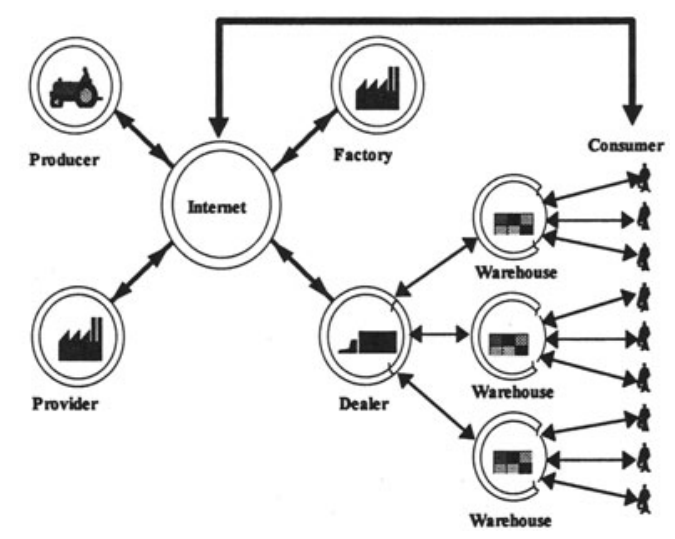

Figure 3 Extended enterprise extranet.

However, taking into consideration the technology evolution on high speed public data communications, compression algorithms and related hardware, a high degree of performance is expected in a near future. The above considerations applies if several private networks (intranets) are to be tied together through the Internet (Figure 3). This structure is called extranet and additional security is mandatory other than firewalls. Encryption algorithms should be used on both ends of any transmission for privacy and authentication. This kind of networks are called Internet Private Virtual Networks (IPVN) and are a commercial research focus at present.

Although the extranet seem to be rather cumbersome as regards security, their communication associated costs are so low that money savings make them an attractive alternative.

\section{QUERIES HINTS}

Client-server data access demands servers to be able to direct the client to the information he needs in a readily and simply way. WEB servers should provide HTML pages containing not only links to the desired topics, but their validation of user rights. 
If multiplatform support is a concern, middle tier applications should be developed. Multiplatform programming languages such as Java and JavaScript should be used as a linking logic.

Besides WEB browser access, other kind of data interchange can be implemented. Peer applications can exchange data on trading activities of the enterprises. This approach enables to implement an automatic data interchange between the several information systems spreaded over the network.

\section{UNDERSTANDING INFORMATION}

Many drawbacks rises from the fact that traditional information management has been paper based, namely :

- High costs and time delays on documentation delivery.

- Information leaks.

- Bypassing of administrative information.

- Maintenance of bureaucratic structures for documentation management.

Many standards have been developed to deal with these problems. Most of them try to translate traditional forms in paper to an electronic equivalent. Perhaps the most popular one at present is EDI (Electronic Data Interchange) (Sokol, 1994).

The development of EDI implies the availability of proper standards for information encoding. Using such standards it is possible for a sender to code the data he wants to export and for the receiver to decode it and to acknowledge the reception. At present there are two standards: the ASCII X12 (ASNSI, 1997) and the UN/EDIFACT (UN/EDIFACT, 1997). The X12 is mainly used domestically in the United States of America, and the UN/EDIFACT is used world wide and is promoted by the United Nations Organisation.

Because of the magnitude and complexity of the universe of all types of EDI messages, the standards above are not complete and are under expansion in order to integrate more business areas. At present, the responsible organisations are working together in order to merge both standards into one.

\section{CONCLUSIONS}

An architecture for the organisation of enterprises in the Extended Enterprise concept, using a client-server model, was presented.

The possibility and potentiality offered by an intranet/extranet for the support of related tasks, such as those proposed here, have been analysed. According to this analysis, realistic implementations can be done without resorting to high investments. 
Security and performance inherent to the utilisation of the Internet for the support of the basic inter-enterprises data communication platform were presented.

Existing standards for electronic data interchange were presented as well as their future evolution.

\section{ACKNOWLEDGEMENTS}

This work was done under the context of the $\mathrm{SCM}+$ project funded by the European Commission INCO-DC program.

\section{REFERENCES}

Browne J. (1996) The extended enterprise - Manufacturing and the value chain The extended enterprise, pp 8- 15

Camarinha-Matos L.M., Afsarmanesh H., Garita C., Lima C. (1997) Towards an Architecture for Virtual Enterprises - The Second World Congress on Intelligent Manufacturing Processes \& Systems, Budapest, Hungary.

Camarinha-Matos L.M., Carelli R., Pellicer J., Martin M. (1997) Towards the Virtual Enterprise in Food Industry. - ISIP'97 OE/IFIP, IEEE International Conference on Integrated and Sustainable Industrial Production, Lisbon, Portugal May 14-14.

SCM+ Beyond Supply Chain Management in Food Industry (1997) Draft Architecture Proposal - Lisbon, Portugal.

Cheswick and Belloin (1994) Firewalls and Internet Security - Repelling the Wily Hacker, Addison-Wesley, ISBN 0-201-63357-4.

Sokol, Phyllis K. (1994) From EDI to Electronic Commerce - Mc.Graw-Hill,Inc. ISBN 0-07-059512-

UN/EDIFACT (1997) http://www.premenos.com/unedifact.

ASNSI web page for ASC X12 (1997) http://www.disa.org/http. 